\title{
Indicadores científicos e tecnológicos em biodiesel na Bahia: panorama sobre grupos de pesquisa do CNPq e pesquisadores da RBTB
}

\author{
Angela Machado Rocha* \\ Marcelo Santana Silva** \\ Cristina Maria Assis Quintela*** \\ Ednildo Andrade Torres****
}

\begin{abstract}
Resumo: O trabalho tem por objetivo propor indicadores científicos e tecnológicos sobre o biodiesel no estado da Bahia, a partir de análise dos Grupos de Pesquisa cadastrados no Conselho Nacional de Desenvolvimento Científico e Tecnológico (CNPq) dos pesquisadores participantes da Rede Brasileira de Tecnologia em Biodiesel (RBTB). Inicialmente, apresenta a relevância da Inovação, assim como o uso dos indicadores científicos e tecnológicos no processo inovativo. Discorre então sobre o biodiesel, relatando sua relevância no contexto atual, assim como seus gargalos tecnológicos no Brasil, enfatizando o Programa Nacional de Produção e Uso do Biodiesel e as perspectivas desse biocombustível na Bahia. A seguir, propõe e analisa indicadores científicos e tecnológicos para o biodiesel na Bahia.
\end{abstract}

Palavras-chave: Biodiesel; Indicadores Científicos; Indicadores Tecnológicos; Bahia.

Classificação JEL: O13; O31; O32; O38.

\footnotetext{
* Doutoranda em energia e ambiente pela Universidade Federal da Bahia (CieNAm-UFBA).

** Doutorando em energia e ambiente pela Universidade Federal da Bahia (CieNAm-UFBA) e professor do Instituto

Federal da Bahia - IFBA - Santo Amaro (antigo CEFET).

*** Professora da Universidade Federal da Bahia (CiNAm-UFBA).

**** Professor da Universidade Federal da Bahia (CieNAm-UFBA).
} 


\section{Introdução}

$\mathrm{Na}$ atual "economia do conhecimento", a geração de valor transferese do material para o conteúdo de conhecimento incorporado aos processos produtivos. Em consequência do desenvolvimento econômico e social estar cada vez mais ancorado na inovação e no conhecimento como agregadores de valor à produção, o tema inovação é palavra de ordem, ocupando lugar central nas esferas política, econômica e social dos países (Tigre, 2006).

Em virtude do crescente reconhecimento da inovação tecnológica como motriz do crescimento econômico e devido à estreita relação existente no setor de Ciência, Tecnologia e Inovação (CT\&I), Winter (2011) assinala que é importante medir a Ciência, Tecnologia e Inovação. Isso se justifica tanto para estimular as investigações e a difusão de conhecimento da CT\&I, como para acompanhar e avaliar as políticas públicas e estratégias de ação de implementação.

Assim, a criação e o uso de indicadores da atividade científica e tecnológica se inserem no contexto atual como valiosos instrumentos para o processo de definição de diretrizes, programas e tomadas de decisão em instâncias governamentais e privadas, já que demonstram as tendências e variáveis do setor que seja foco do objeto de análise.

Por outro lado, no atual estágio mundial de crescimento econômico, a demanda por energia tem crescido aceleradamente. Entretanto, 80\% ou mais da energia utilizada é proveniente de combustíveis fósseis, principalmente o petróleo. Uma vez que as reservas exploráveis desse recurso são finitas, esse estoque se extinguirá. O esgotamento das reservas petrolíferas, aliado às preocupações com a degradação ambiental gerada pela sua queima, tem motivado vários países a buscar soluções para suprir suas necessidades energéticas pela substituição do petróleo por biodiesel, o que se justificada pela sua expressiva capacidade de redução da emissão de poluentes e de gases de efeito estufa desse biocombustível (Portella, 2008).

Os dados do Balanço Energético Nacional (MME, 2012) indicam que o Brasil possui $44,1 \%$ de sua matriz energética exemplar composta por fontes renováveis, o que contrasta significativamente com a média mundial de cerca de $13,3 \%$.

Atento a essas questões de necessidade de mudança da matriz energética e de busca por inovações para impulsionar o desenvolvimento econômico sustentável do estado, o Governo da Bahia aborda essas questões no seu Planejamento Plurianual Anual (PPA 2012-2015), plano no qual o governo define as diretrizes, os programas e as ações para os próximos quatro anos. Dessa maneira, o eixo II "Desenvolvimento Sustentável e Infraestrutura para o Desenvolvimento" estabelece programas como o de "estimular o crescimento da produção de energia no Estado, diversificando a composição da matriz, com ênfase no aumento da participação das energias renováveis e promoção de maior eficiência energética". Também objetiva "tornar a Bahia referência nacional em Pesquisa e Desenvolvimento (P\&D), inovação e transferência de tecnologia nas áreas de energia e ambiente" (BAHIA, 2011). 
Nesse contexto, o presente trabalho propõe e apresenta indicadores, analisando e discutindo os resultados encontrados nos aspectos relacionados ao desenvolvimento científico e tecnológico em biodiesel na Bahia, o que contribui para um melhor entendimento das perspectivas de inovação do biocombustível no Estado.

\section{Metodologia}

Os indicadores científicos e tecnológicos analisados foram extraídos através de dados disponíveis no Diretório dos Grupos de Pesquisa no Brasil do Conselho Nacional de Desenvolvimento Científico e Tecnológico (DGP/CNPq), da Rede Brasileira Tecnológica do Biodiesel (RBTB) e da Plataforma Lattes do Conselho Nacional de Desenvolvimento Científico e Tecnológico (CNPq).

O DGP/CNPq constitui-se em bases de dados que contém informações sobre os grupos de pesquisa em atividade no País. São inventariados apenas os que estão localizados em universidades, instituições isoladas de ensino superior, institutos de pesquisa científica, institutos tecnológicos e laboratórios de pesquisa e desenvolvimento de empresas estatais ou ex-estatais, sendo excluídos os grupos localizados nas empresas do setor produtivo. Cada grupo é situado no espaço (região, UF e instituição) e no tempo. As informações do Diretório são atualizadas continuamente e o CNPq realiza censos bi-anuais, que são fotografias dessa base corrente (CNPq, 2011). Para este trabalho foi pesquisado até período de 2011. Espera-se que até o final de 2013 o banco de dados esteja atualizado.

No DGP/CNPq, foram buscados indicadores sobre os recursos humanos constituintes dos grupos (pesquisadores, estudantes e técnicos). Investigou-se sobre ao quantitativo de pessoas alocadas em cada grupo, especialidades do conhecimento; à produção científica e tecnológica; e a interação com o setor produtivo. Para tanto, buscou-se na base textual do DGP/CNPq, os grupos de pesquisa que possuíssem a palavra-chave "biodiesel", aplicando-se como filtro de seleção o estado da Bahia.

Adicionalmente, foram investigados os pesquisadores de Instituições Científicas e Tecnológicas (ICTs) do estado da Bahia, com artigos publicados nos anais dos Congressos da Rede Brasileira Tecnológica do Biodiesel (RBTB). Nessa busca, foram excluídos os estudantes de Iniciação Científica, Pós-graduandos e Técnicos.

Posteriormente, os dados extraídos da RBTB foram cruzados e comparados aos encontrados na busca realizada no DGP/CNPq. Foram também investigados os dados disponíveis na base de dados de currículos Lattes dos Pesquisadores em questão.

Dentre os diversos indicadores empregados na análise da produção científica, foram analisadores Indicadores de Produção Científica, construídos pela contagem da produção bibliográfica, por Instituição de Ciência e Tecnologia (ICT), área de conhecimento, contingente de pesquisadores. Para os de Produção 
Tecnológica, utilizou-se a contagem de produção tecnológica assim como a interação com o setor produtivo.

\section{Indicadores Científicos e Tecnológicos}

Com a crescente relevância do papel da Inovação, Ciência e da Tecnologia como determinantes do desenvolvimento econômico, tornou-se cada vez mais evidente a necessidade de medição das taxas de produtividade dos centros de pesquisa e dos investigadores individuais, para a identificação daquelas instituições e áreas com maiores potencialidades e para o estabelecimento das prioridades no momento da alocação de recursos públicos (Liberal, 2005).

Assim, a Organização Européia de Cooperação Econômica (OCDE) apresenta, em 1963, uma "Proposta de um Sistema Padrão para Avaliação em Pesquisa e Desenvolvimento", que origina o Manual Frascati. O Manual Frascati passou por revisões ao longo do tempo. Atualmente, os indicadores compreendem os dispêndios realizados, assim como o pessoal dedicado às atividades de C\&T (OCDE, 2005).

Em 1992, surgiu a $1^{a}$ edição do Manual de Oslo, com Proposta de Diretrizes para Coleta e Interpretação de Dados sobre Inovação Tecnológica. Em 1997 e 2005 são lançadas as respectivas $2^{a}$ e $3^{a}$ edição do referido Manual.

A série de Manuais metodológicos da OCDE é conhecida como a "Família Frascati”, compreendendo os seguintes assuntos: P\&D (Manual Frascati), o balanço de pagamentos de tecnologia e estatísticas de inovação (Manual de Oslo); o uso de estatísticas sobre patentes como indicadores de Ciência e Tecnologia (Patent Manual - Manual de Patentes) e recursos humanos dedicados à ciência e tecnologia (Manual Camberra).

Apesar das limitações metodológicas, os manuais citados são largamente utilizados tanto pelos formuladores de políticas como para comparação dos dados estatísticos em nível internacional.

\section{Biodiesel}

\subsection{Conceito}

Por definição técnica, biodiesel é um combustível composto de monoalquilésteres de ácidos graxos de cadeia longa, derivado de óleos vegetais ou de gorduras animais e designado B100. Biodiesel é um combustível biodegradável derivado de fontes renováveis, que pode ser obtido por diferentes processos tais como o craqueamento, a esterificação ou pela transesterificação. Pode ser produzido a partir de gorduras animais ou de óleos vegetais. O biodiesel substitui total ou parcialmente o óleo diesel de petróleo em motores ciclo diesel automotivos ou estacionários. Pode ser usado puro ou misturado ao diesel em diversas proporções (Quintella et al., 2009). 


\subsection{Biodiesel no Brasil}

Em 13 de janeiro de 2005, foi publicada a Lei 11.097, que dispõe sobre a introdução do biodiesel na matriz energética brasileira. Define o biodiesel como "biocombustível derivado de biomassa renovável para uso em motores a combustão interna com ignição por compressão ou, conforme regulamento para geração de outro tipo de energia, que possa substituir parcial ou totalmente combustíveis de origem fóssil" (Brasil, 2005).

A Lei obrigou que todo o diesel utilizado em território nacional fosse adicionado $2 \%$ de biodiesel (B2) em sua composição. A fase autorizativa do PNPB corresponde ao período de 2005-2007. A partir de 2008, torna-se obrigatória a adição do biodiesel ao diesel. Esta mistura sofreu aumentos gradativos, para B3 em março de 2008, B4 em julho de 2009 e B5, desde janeiro de 2010 (MCT, 2011).

O parque industrial para a produção de biodiesel no Brasil cresceu em ritmo acelerado. Em 2005, início da implementação do PNPB, eram apenas oito

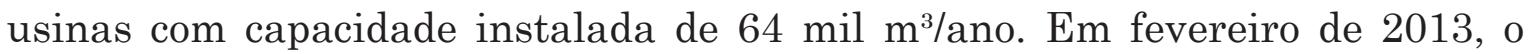
número de usinas autorizadas para produzir e comercializar biodiesel através dos leilões chegou a 56 com capacidade instalada de produção de $6.853 \mathrm{mil} \mathrm{m}^{3} / \mathrm{ano}$, conforme o gráfico 1 (MME, 2013).

\section{Gráfico 1 - Produção e Capacidade Instalada de Produção de Biodiesel no Brasil}

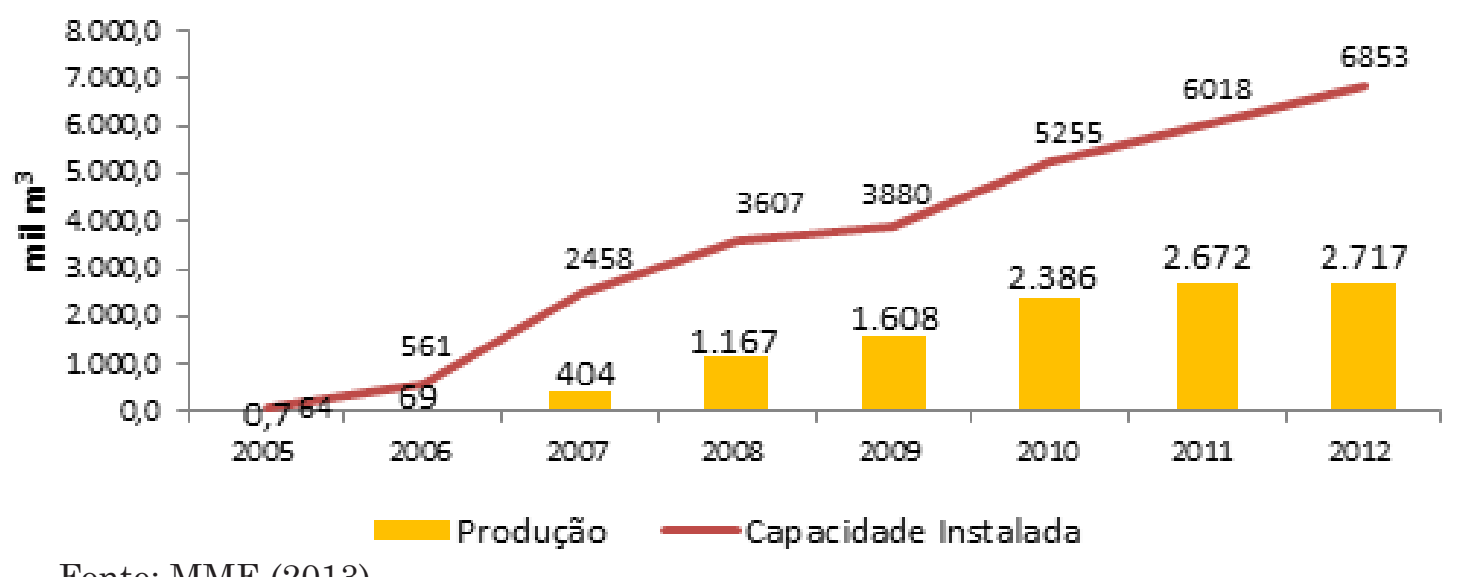

Fonte: MME (2013)

Observa-se que de 2005 a 2012 houve crescimento na produção de biodiesel, em virtude da obrigatoriedade da regulamentação imposta no país. Nos últimos três anos, a média foi de 2,5 bilhões de litros/ano, haja vista que essa expansão da produção industrial foi mais expressiva em seus cinco primeiros anos.

Com relação às matérias-primas utilizadas para produzir biodiesel em 2012, têm-se: a soja representando $75,2 \%$, seguida pelo sebo bovino (17,2\%), algodão $(4,5 \%)$, outros materiais graxos $(3 \%)$ e outras matérias-primas $(0,1 \%)$ (MME, 2013). 


\subsection{O cenário do biodiesel no Nordeste e na Bahia}

No atual cenário, dos nove Estados do Nordeste, somente dois estão produzindo biodiesel: Bahia e Ceará. Esses estados responderam por cerca de $10 \%$ do volume de produção nacional (ANP, 2013). O gráfico 2 a seguir mostra a produção de biodiesel dos estados nordestinos no período de 2005 a 2012.

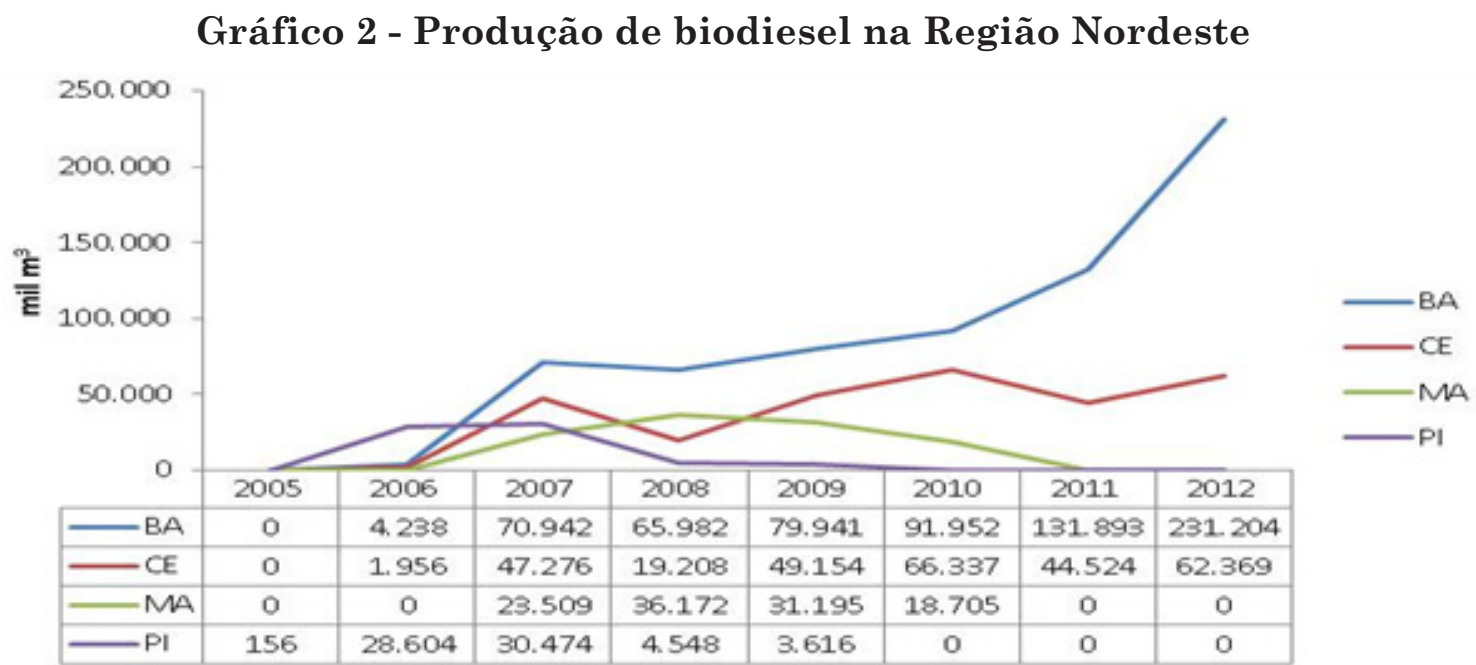

Fonte: ANP (2013)

Apesar desse volume, a região apresenta um déficit de $163.110 \mathrm{~m}^{3} \mathrm{de}$ biodiesel para atender a mistura dos $9.133 .665 \mathrm{~m}^{3}$ de óleo diesel consumido na região em 2012 (ANP, 2013).

A Bahia possui quatro usinas para comercialização de biodiesel, perfazendo uma capacidade instalada de $502.7011 \mathrm{~m}^{3} /$ ano. A Petrobras Biocombustível (PBIO), localizada em Candeias, com 217,2 mil mªno; a V-Biodiesel, localizada em Iraquara, com 129,6 mil m³/ano; a Comanche, localizada em Simões Filho, com 120,6 mil mªno; e a Biobrax, localizada em Una com 35,2 $\mathrm{mil} \mathrm{m}^{3} / \mathrm{ano}$ (ANP, 2013).

\subsection{Pesquisas em biodiesel no Brasil}

O Brasil vem desenvolvendo pesquisas sobre biodiesel há algumas décadas, inclusive registrando patente sobre o processo de produção de combustível, em 1980 (PORTAL DO BIODIESEL, 2006).

Em relação ao PNPB, o módulo de Desenvolvimento Tecnológico é coordenado pelo Ministério de Ciência e Tecnologia e Inovação (MCT), o qual abrange a constituição da Rede Brasileira de Tecnologia de Biodiesel (RBTB), cujo escopo é a consolidação de um sistema gerencial de articulação dos diversos atores envolvidos na pesquisa, no desenvolvimento e na produção de biodiesel, permitindo assim a convergência de esforços e otimização de investimentos públicos. A RBTB é constituída por subredes temáticas, conforme quadro 1 . 
Desde 2006, a RBTB organiza Congressos, onde se verifica crescente produção científica.

\begin{tabular}{|c|c|}
\hline MATÉRIA PRIMA & $\begin{array}{l}\text { Sistema de produção e obtenção de matérias primas de origem } \\
\text { vegetal e animal; zoneamento pedoclimático; variedades vegetais e } \\
\text { oleaginosas; OGRs (óleos e gorduras residuais); microorganismos e algas; } \\
\text { economia e modelagem de sistemas; processamento e transformação. }\end{array}$ \\
\hline $\begin{array}{l}\text { ESTABILIDADE, } \\
\text { ARMAZENAMENTO } \\
\text { E PROBLEMAS } \\
\text { ASSOCIADOS }\end{array}$ & $\begin{array}{l}\text { Estudos de estabilidade, formas de armazenamento do biodiesel e das } \\
\text { misturas (biodiesel \& diesel); avaliação e desenvolvimento de aditivos; } \\
\text { vida útil; avaliação das condições ideais de condicionamento do produto . }\end{array}$ \\
\hline $\begin{array}{l}\text { CARACTERIZAÇÃO } \\
\text { E CONTROLE DE } \\
\text { QUALIDADE }\end{array}$ & $\begin{array}{l}\text { Caracterização do óleo in natura, do combustível e suas misturas } \\
\text { (biodiesel \& diesel), oriundo de diversas matérias primas, assim } \\
\text { como o desenvolvimento de metodologias para análise e controle } \\
\text { de qualidade, visando maior praticidade e economicidade. }\end{array}$ \\
\hline COPRODUTOS & $\begin{array}{l}\text { Estudos quanto ao destino e uso dos coprodutos } \\
\text { (glicerina, } \\
\text { torta, farelo, etc.) para que seja garantida a agregação de valor } \\
\text { e criadas outras fontes de renda para os produtores de biodiesel. }\end{array}$ \\
\hline PRODUÇÃO & $\begin{array}{l}\text { Desenvolvimento (otimização) de tecnologia para produção de } \\
\text { biodiesel em laboratório e em escalas adequadas às produções locais } \\
\text { de óleo, de forma a garantir qualidade e economicidade das plantas. }\end{array}$ \\
\hline
\end{tabular}

Fonte: RBTB (2011)

Também, em consulta à base de dados do DGP/CNPq, verifica-se a existência de 330 grupos de pesquisa no Brasil com a temática biodiesel, como palavra-chave.

\subsection{O programa de biodiesel na Bahia}

A partir de 1970, a Bahia experimentou expressivo crescimento econômico, impactando tanto a oferta quanto a demanda de energia. A entrada em operação do Centro Industrial de Aratu (CIA), do Complexo Petroquímico de Camaçari (COPEC) e do Pólo de Papel e Celulose, este último a partir de 1992, fez com que a demanda energética estadual concentra-se nas indústrias Metalúrgica, Química e de Papel e Celulose.

A Bahia vem estimulando o crescimento da produção de energia no Estado, diversificando a composição da matriz, com ênfase no aumento da participação das energias renováveis e promoção de maior eficiência energética.

O Estado possui disponibilidade de áreas e condições edafoclimáticas favoráveis ao cultivo de oleaginosas aptas à produção do biodiesel, concentrando $80 \%$ da produção de mamona do país. A agricultura baiana também apresenta expressivos resultados no cultivo do algodão, do dendê, da soja e do girassol, que são, igualmente, grandes fontes de óleo para o biodiesel.

Entretanto, em relação ao setor do Biodiesel, os programas na Bahia são recentes. Em 2003, foi criado o Programa de Biodiesel da Bahia - Probiodiesel Bahia, coordenado pela Secretaria de Ciência Tecnologia e Inovação (SECTI), 
que se constituiu, na fase de estruturação do programa, na criação da rede de relacionamento e da construção da carteira de projetos. Nesse período, foi criada a Rede Baiana de Biocombustíveis - RBB, com o objetivo de promoção do desenvolvimento tecnológico, a partir da interação entre os diversos agentes (Silva et al, 2010).

Em 2007, foi lançado o Programa Produção e Uso de Combustíveis na Bahia - Biosustentável, conhecido por BAHIABIO, com a finalidade de gerir e fomentar ações, desenvolvimento, aplicações e uso de biomassa no território baiano, bem como implantar no Estado o biodiesel como um biocombustível adicional à matriz energética, além de estimular pesquisas relacionadas ao Programa.

\section{Resultados Encontrados}

Na base textual do DGP/CNPq foram identificados 26 grupos de pesquisa com a palavra-chave "biodiesel", aplicando-se como filtro de seleção o estado da Bahia, conforme quadro 2 abaixo.

Quadro 2 - Grupos de Pesquisa de ICTs da Bahia em Biodiesel, segundo o DGP/CNPq

\begin{tabular}{|l|c|c|c|c|c|c|c|}
\hline NOME DO GRUPO & ICT & ANO & ÁREA & PQ & E & T & SP \\
\hline $\begin{array}{l}\text { Biodiesel e Desenvolvimento } \\
\text { Sustentável }\end{array}$ & UESB & 2007 & Agronomia & 10 & 2 & 0 & 0 \\
\hline Bioenergia & FTC & 2009 & Química & 9 & 8 & 0 & 6 \\
\hline Bioenergia e meio ambiente & UESC & 2000 & Química & 11 & 14 & 1 & 0 \\
\hline Catalise e Ambiente & UNIFACS & 2004 & Eng.Química & 7 & 17 & 17 & 0 \\
\hline $\begin{array}{l}\text { Centro de Tecnologia e de Gestão } \\
\text { ao Desenvolvimento Regional - } \\
\text { CETEG }\end{array}$ & UEFS & 2006 & Administração & 10 & 5 & 0 & 0 \\
\hline Cinética e Dinâmica Molecular & UFBA & 1994 & Química & 4 & 23 & 6 & 1 \\
\hline $\begin{array}{l}\text { Conservação e Manejo } \\
\text { Sustentável da Biodiversidade }\end{array}$ & EBDA & 2002 & Biologia & 21 & 11 & 8 & 0 \\
\hline Engenharia Bioquímica & UEFS & 2004 & Eng.Química & 8 & 8 & 0 & 0 \\
\hline $\begin{array}{l}\text { GEMAC - Grupo de Estudos em } \\
\text { Materiais de Construção }\end{array}$ & UFBA & 1997 & Eng.Civil & 11 & 6 & 6 & 2 \\
\hline $\begin{array}{l}\text { GFALEVALE - Grupo de Fontes } \\
\text { Alternativas de Energia do Vale } \\
\text { do São Francisco }\end{array}$ & UNIVASF & 2008 & Eng.Elétrica & 6 & 5 & 1 & 0 \\
\hline $\begin{array}{l}\text { Grupo de Energia e Ciência dos } \\
\text { Materiais }\end{array}$ & UFBA & 2004 & Química & 6 & 24 & 1 & 0 \\
\hline $\begin{array}{l}\text { Grupo de Estudos em } \\
\text { Fenômenos de Superfície }\end{array}$ & UEFS & 2004 & Química & 2 & 1 & 0 & 0 \\
\hline $\begin{array}{l}\text { Grupo de Pesquisa e Inovação } \\
\text { em Química }\end{array}$ & IFBA & 2006 & Química & 19 & 13 & 0 & 0 \\
\hline $\begin{array}{l}\text { Grupo de Pesquisa em } \\
\text { Biodiesel, Catálise e Ambiental } \\
\text {-GGPBCAT }\end{array}$ & IFBA & 2009 & Química & 6 & 1 & 1 & 1 \\
\hline
\end{tabular}




\begin{tabular}{|c|c|c|c|c|c|c|c|}
\hline NOME DO GRUPO & ICT & ANO & ÁREA & $\mathbf{P Q}$ & $\mathbf{E}$ & $\mathbf{T}$ & SP \\
\hline $\begin{array}{l}\text { Grupo de Pesquisa e m Energia e } \\
\text { Materiais }\end{array}$ & IFBA & 2009 & Eng.Química & 16 & 6 & 1 & 0 \\
\hline $\begin{array}{l}\text { Grupo de Pesquisa em Meio } \\
\text { Ambiente }\end{array}$ & SENAI & 2003 & Eng.Química & 7 & 3 & 9 & 0 \\
\hline $\begin{array}{l}\text { Grupo de Pesquisa em Química } \\
\text { Analítica }\end{array}$ & UFBA & 1997 & Química & 9 & 27 & 0 & 0 \\
\hline $\begin{array}{l}\text { Grupo Interdisciplinar de } \\
\text { Química-GIQ }\end{array}$ & UFRB & 2009 & Química & 7 & 14 & 0 & 0 \\
\hline $\begin{array}{l}\text { IDEIA - Grupo de Investigação, } \\
\text { DEsenvolvimento e Inovação } \\
\text { Analíticas }\end{array}$ & UFBA & 2009 & Química & 6 & 13 & 1 & 1 \\
\hline $\begin{array}{l}\text { Laboratório de Automação e } \\
\text { Instrumentação Analítica }\end{array}$ & UFRB & 2007 & Química & 6 & 22 & 1 & 0 \\
\hline $\begin{array}{l}\text { Núcleo de Biotecnologia - } \\
\text { NuBiotec }\end{array}$ & FTC & 2005 & Farmácia & 5 & 8 & 2 & 2 \\
\hline $\begin{array}{l}\text { Núcleo de Pesquisa em } \\
\text { Energética Química e } \\
\text { Petroquímica - NEQP }\end{array}$ & UNIFACS & 2000 & Eng.Química & 9 & 24 & 6 & 0 \\
\hline $\begin{array}{l}\text { Petróleo, Gás Natural e } \\
\text { Biocombustíveis }\end{array}$ & UFBA & 2010 & Eng.Química & 5 & 9 & 0 & 0 \\
\hline $\begin{array}{l}\text { Produção e Tecnologia } \\
\text { Sustentável no Bioma Cerrado }\end{array}$ & UNEB & 2011 & $\begin{array}{l}\text { Ciência e } \\
\text { Tecnol. de } \\
\text { Alimentos }\end{array}$ & 15 & 31 & 4 & 0 \\
\hline $\begin{array}{l}\text { Química Ambiental e } \\
\text { Desenvolvimento Sustentável }\end{array}$ & UFBA & 2006 & Química & 12 & 12 & 0 & 0 \\
\hline $\begin{array}{l}\text { Química e Bioquímica de } \\
\text { Alimentos }\end{array}$ & UESB & 2009 & $\begin{array}{l}\text { Ciência e } \\
\text { Tecnol. de } \\
\text { Alimentos }\end{array}$ & 5 & 12 & 0 & 0 \\
\hline
\end{tabular}

Legendas: (ICT): Instituições de Ciência e Tecnologia

UFBA- Universidade Federal da Bahia

UFRB- Universidade Federal do Recôncavo.

UNIVASF- Universidade Federal do Vale do São Francisco

IFBA - Instituto Federal da Bahia

UNEB- Universidade do Estado da Bahia

UESB- Universidade Estadual do Sudoeste da Bahia

UESC- Universidade Estadual de Santa Cruz

UEFS - Universidade Estadual de Feira de Santana

UNIFACS- Universidade Salvador

EBDA - Empresa Baiana de Desenvolvimento Agrícola

FTC -Faculdade de Tecnologia e Ciências

SENAI- Serviço Nacional de Aprendizagem Industrial

(Ano): Ano de Formação do Grupo (Área): Área de Concentração do Grupo

(PQ): Número de Pesquisadores

(E): Número de Estudantes

(T):Número de Técnicos

(SP): Interação com o Setor Produtivo

A tabela 1 apresenta indicadores de C\&T nos aspectos de Produção Científica e Tecnológica dos Grupos em questão.

$\mathrm{Na}$ construção da tabela 1, foram consideradas apenas a Produção Bibliográfica (PB) e Produção Técnica (PT) declaradas pelos líderes de cada grupo investigado no período de 2008-2010. Foram contabilizados os dados somente a partir do ano de formação do grupo em questão, sendo desprezadas as produções anteriores a esse período.

É importante lembrar que, de acordo com o CNPq, a produção científica 
e tecnológica de um grupo é a soma da produção existente nos currículos Lattes dos pesquisadores e estudantes que participam do grupo, admitida a dupla ou múltipla contagem que ocorre devido às co-autorias entre participantes do grupo.

Tabela 1 - Indicadores de C \& T dos Grupos de Pesquisa de ICTs da Bahia em Biodiesel, segundo o DGP/CNPq

\begin{tabular}{|c|c|c|c|c|c|c|}
\hline NOME DO GRUPO & $\begin{array}{c}\mathrm{PB} \\
2008\end{array}$ & $\begin{array}{c}\mathrm{PB} \\
2009\end{array}$ & $\begin{array}{c}\text { PB } \\
2010\end{array}$ & $\begin{array}{c}\text { PT } \\
2008\end{array}$ & $\begin{array}{c}\text { PT } \\
2009\end{array}$ & $\begin{array}{c}\text { PT } \\
2010\end{array}$ \\
\hline Biodiesel e Desenvolvimento Sustentável & 8 & 8 & 9 & 4 & 4 & 8 \\
\hline Bioenergia & 0 & 8 & 4 & 0 & 0 & 0 \\
\hline Bioenergia e Meio Ambiente & 5 & 10 & 4 & 4 & 3 & 0 \\
\hline Catalise e Ambiente & 8 & 18 & 10 & 4 & 2 & 2 \\
\hline $\begin{array}{l}\text { Centro de Tecnologia e de Gestão Ao } \\
\text { Desenvolvimento Regional - CETEG }\end{array}$ & 1 & 0 & 0 & 0 & 0 & 0 \\
\hline Cinética e Dinâmica Molecular & 21 & 21 & 26 & 35 & 22 & 35 \\
\hline $\begin{array}{l}\text { Conservação e Manejo Sustentável da } \\
\text { Biodiversidade }\end{array}$ & 2 & 4 & 3 & 0 & 2 & 0 \\
\hline Engenharia Bioquímica & 2 & 1 & 0 & 0 & 0 & 0 \\
\hline $\begin{array}{l}\text { GEMAC - Grupo de Estudos em Materiais de } \\
\text { Construção }\end{array}$ & 1 & 1 & 2 & 0 & 4 & 0 \\
\hline $\begin{array}{l}\text { GFALEVALE - Grupo de Fontes Alternativas } \\
\text { de Energia do Vale do São Francisco }\end{array}$ & 1 & 5 & 4 & 2 & 0 & 1 \\
\hline Grupo de Energia e Ciência dos Materiais & 14 & 18 & 16 & 2 & 3 & 0 \\
\hline Grupo de Estudos em Fenômenos de Superfície & 1 & 2 & 1 & 9 & 0 & 0 \\
\hline Grupo de Pesquisa e Inovação em Química & 11 & 5 & 6 & 5 & 11 & 24 \\
\hline $\begin{array}{l}\text { Grupo de Pesquisa em Biodiesel, Catálise e } \\
\text { Ambiental GPBCAT }\end{array}$ & 0 & 2 & 0 & 1 & 0 & 0 \\
\hline Grupo de Pesquisa em Energia E Materiais & 0 & 2 & 0 & 0 & 0 & 0 \\
\hline Grupo de Pesquisa em Meio Ambiente & 0 & 1 & 1 & 2 & 2 & 0 \\
\hline Grupo de Pesquisa em Química Analítica & 12 & 7 & 0 & 0 & 1 & 0 \\
\hline Grupo Interdisciplinar de Química-GIQ & 0 & 3 & 1 & 4 & 4 & 10 \\
\hline $\begin{array}{l}\text { IDEIA - Grupo de Investigação, } \\
\text { DEsenvolvimento e Inovação Analíticas }\end{array}$ & 0 & 11 & 8 & 16 & 5 & 6 \\
\hline $\begin{array}{l}\text { Laboratório de Automação e Instrumentação } \\
\text { Analítica }\end{array}$ & 1 & 6 & 1 & 2 & 0 & 0 \\
\hline Núcleo de Biotecnologia - NuBiotec & 4 & 6 & 1 & 7 & 0 & 7 \\
\hline $\begin{array}{l}\text { Núcleo de Pesquisa em Energética Química e } \\
\text { Petroquímica - NEQP }\end{array}$ & 8 & 18 & 10 & 4 & 2 & 2 \\
\hline Petróleo, Gás Natural e Biocombustíveis & 0 & 0 & 3 & 0 & 0 & 0 \\
\hline $\begin{array}{l}\text { Produção e Tecnologia Sustentável no Bioma } \\
\text { Cerrado }\end{array}$ & 0 & 0 & 0 & 0 & 0 & 0 \\
\hline $\begin{array}{l}\text { Química Ambiental e Desenvolvimento } \\
\text { Sustentável }\end{array}$ & 3 & 4 & 1 & 3 & 2 & 3 \\
\hline Química e Bioquímica de Alimentos & 0 & 2 & 7 & 0 & 1 & 2 \\
\hline
\end{tabular}

Legenda: PB - Produção Bibliográfica; PT - Produção Técnica 
Em relação aos autores de ICTs da Bahia que publicaram nos diversos congressos da RBTB, foram identificados 23 autores com artigos em diversas áreas temáticas, conforme quadro 3 . O referido quadro também apresenta os autores da RBTB em relação ao pertencimento (ou não) dos $\mathrm{DGP} / \mathrm{CNPq}$ em biodiesel de ICTs da Bahia já investigados.

Quadro 3 - Autores de ICTs da Bahia, temas e grupos de pesquisa em biodiesel (DGP/CNPq

\begin{tabular}{|c|c|c|}
\hline AUTORES & TEMAS & GRUPO DE PESQUISA/CNPq \\
\hline Cristina M. Quintella & $\mathrm{U}, \mathrm{CP}, \underset{\mathrm{S}}{\mathrm{DS}}, \mathrm{CQ}$ & Cinética e Dinâmica Molecular \\
\hline Ednildo Andrade Torres & $\mathrm{P}, \mathrm{DS}, \mathrm{U}$ & Outros \\
\hline Marilena Meira & $\mathrm{CQ}$ & Cinética e Dinâmica Molecular \\
\hline Leonardo S. G. Teixeira & $\mathrm{P}, \mathrm{U}$ & $\begin{array}{l}\text { IDEIA - Grupo de Investigação, } \\
\text { Desenvolvimento e Inovação } \\
\text { Analíticas }\end{array}$ \\
\hline Maria Bernadete N..Leite & $\mathrm{DS}, \mathrm{S}$ & Outros \\
\hline Silvio A. B. Vieira de Melo & $\mathrm{P}$ & Outros \\
\hline Iracema A. Nascimento & DS, S & Outros \\
\hline Solange Andrade Pereira & $\mathrm{DS}, \mathrm{S}$ & Outros \\
\hline Heloysa M. C. Andrade & $\mathrm{P}$ & Outros \\
\hline Telma Côrtes Q. de Andrade & $\mathrm{DS}, \mathrm{S}$ & Outros \\
\hline Luciene S. de Carvalho & $\mathrm{U}$ & $\begin{array}{l}\text { Núcleo de Pesquisa em Energética } \\
\text { Química e Petroquímica - NEQP }\end{array}$ \\
\hline Maria das Graças Korn & $\mathrm{DS}$ & $\begin{array}{l}\text { Grupo de Pesquisa em Química } \\
\text { Analítica }\end{array}$ \\
\hline Lusimar G. Fernandez & $\mathrm{A}$ & Outros \\
\hline José Adolfo de Almeida Neto & $\mathrm{DS}, \mathrm{CP}, \mathrm{S}$ & Bioenergia e meio ambiente \\
\hline Rosenira Serpa da Cruz & $\mathrm{P}, \mathrm{CP}$ & Bioenergia e meio ambiente \\
\hline Kil Jim Park & $\mathrm{A}$ & $\begin{array}{l}\text { Centro de Tecnologia e de Gestão ao } \\
\text { Desenvolvimento Regional - CETEG }\end{array}$ \\
\hline Djane S. de Jesus & $\mathrm{CP}$ & Outros \\
\hline Alexandre S. Machado & $\mathrm{CP}$ & Outros \\
\hline Rui Carlos A. Lima & $\mathrm{CP}$ & Não filiado a Grupo de Pesquisa \\
\hline Ana Maria de Oliveira & $\mathrm{P}, \mathrm{CP}$ & Bioenergia e meio ambiente \\
\hline Mônica de Moura Pires & $\mathrm{P}, \mathrm{S}$ & Bioenergia e meio ambiente \\
\hline Marcia Cristina C. Veloso & $\mathrm{CP}$ & Outros \\
\hline
\end{tabular}

Legendas: A- Agricultura; P - Produção; S - Sustentabilidade; U - Uso; CP - Coproduto; CQ Caracterização e Qualidade; MP - Matéria Prima; DS - Desenvolvimento Sustentável

\section{Considerações sobre os Resultados Encontrados}

Segundo o DGP/CNPq, há 26 grupos de pesquisa em biodiesel na Bahia, com anos de criação variando de 1994 a 2011, conforme apresentado na figura 3. 
Figura 3 - Grupos de Pesquisa por Ano de Fundação

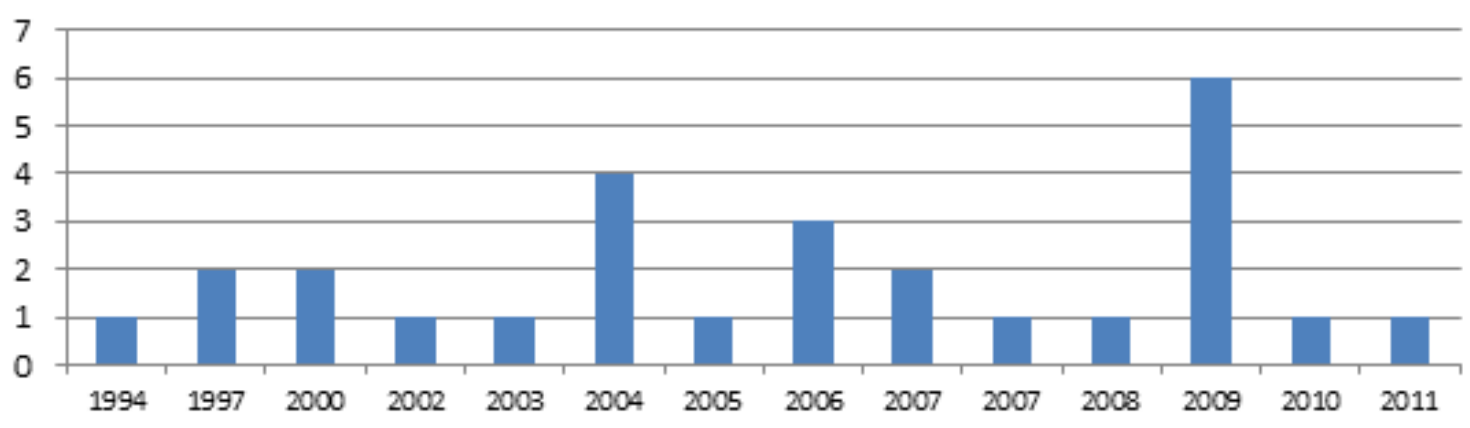

Fonte: Elaboração própria

Observam-se períodos de pico nos anos de 2004, ano de criação da RBTB, e no ano de 2009, ano seguinte da obrigatoriedade do cumprimento da Lei 11097/2005. Também se pode atribuir ao crescimento a contribuição dos programas para o fomento em biodiesel na Bahia, o Probiodiesel Bahia (2003) e o BAHIABIO (2007).

Comparando os autores da RBTB, que também são pesquisadores do DPQ/CNPq em biodiesel na Bahia, observa-se que 11 dos 23 autores não pertencem aos grupos de pesquisa investigados.

Isso pode ser justificado, pois, nesse presente trabalho, no mapeamento dos grupos do DGP/CNPq utilizou-se a palavra-chave "biodiesel". O DGP/CNPq suporta até 4 palavras-chaves para identificar um grupo. Assim, é provável que os 11 pesquisadores pertençam a grupos de pesquisa que não possuam a palavra-chave "biodiesel".

A instituição com maior número de grupos de pesquisa é a Universidade Federal da Bahia (UFBA), que é também a mais antiga e com maior histórico em pesquisas.

Em seguida, vem o Instituto Federal da Bahia (IFBA), com forte tradição em cursos tecnológicos na área de Química, conforme figura 4 a seguir.

A Universidade Federal do Recôncavo (UFRB), terceira no ranking de ICTs em pesquisa em biodiesel, foi estabelecida em 2005, por desmembramento da Escola de Agronomia da Universidade Federal da Bahia.

Os grupos concentram-se nas áreas de Química e Engenharia Química, conforme figura 5 abaixo. Isso pode ser explicado pelo fato de as verbas do PNPB nas temáticas de Caracterização/ Controle e Produção serem as que possuem mais valores para pesquisas. 


\section{Figura 4 - Grupos do DGP/ CNPq por ICTs}

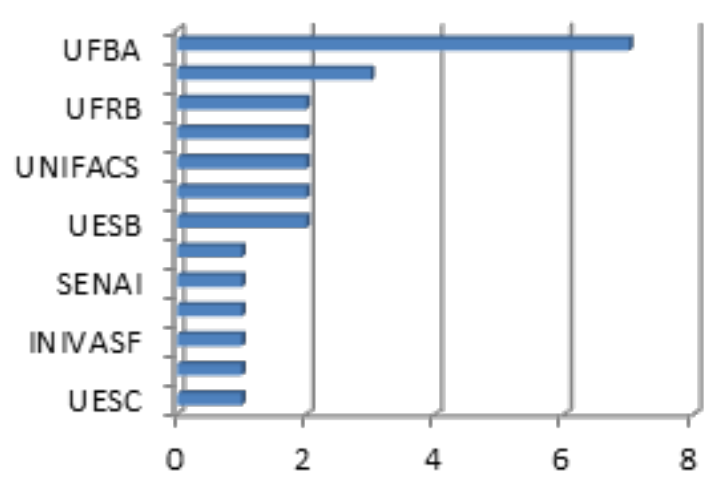

Fonte: Elaboração própria

\author{
Figura 5 - Área de Concentração \\ dos Grupos de Pesquisa
}

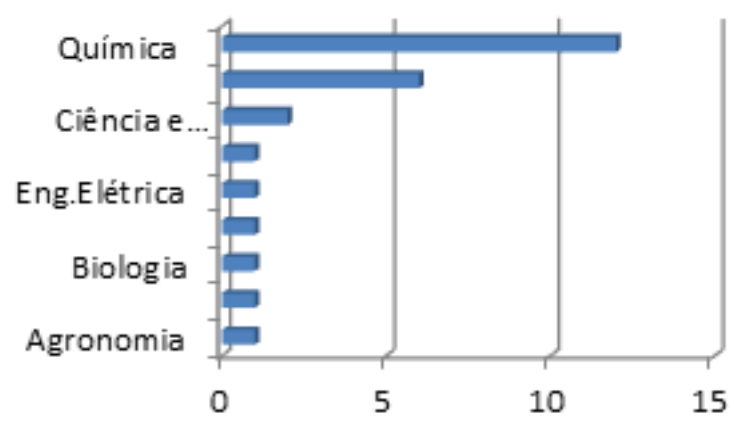

Fonte: Elaboração própria

Os grupos do DGP/CNPq investigados possuem, no total, 232 pesquisadores, 319 estudantes e 65 técnicos.

Os pesquisadores pertencem a diversas áreas do conhecimento, uma vez que o tema biodiesel assim como o PNPB é essencialmente interdisciplinar, com vertentes.

A figura 6 representa o número de pesquisadores alocados por áreas dos Grupos de Pesquisa investigados, onde novamente nota-se a concentração nas áreas de Química e Engenharia Química. Os temas mais freqüentes são apresentados na figura 7 .

Figura 6 - Quantidade de pesquisadores alocados nos grupos de pesquisa

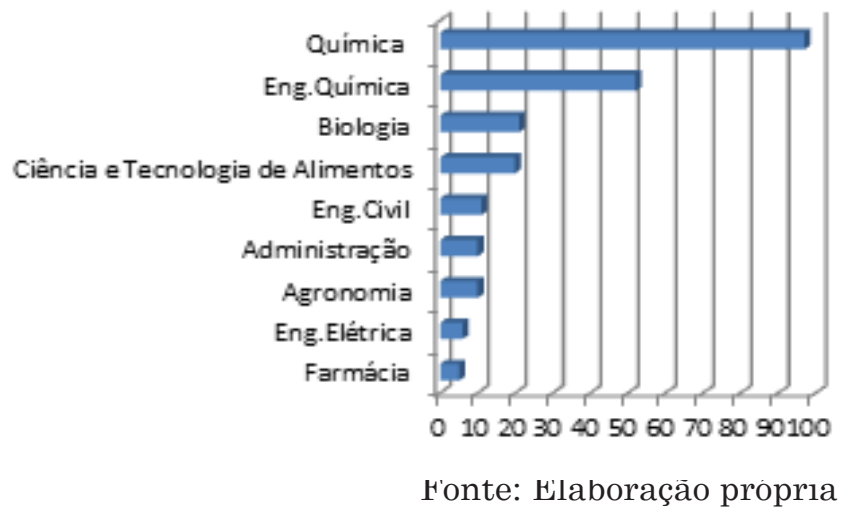

Figura 7 - Temas frequentes de pesquisadores na RBTB

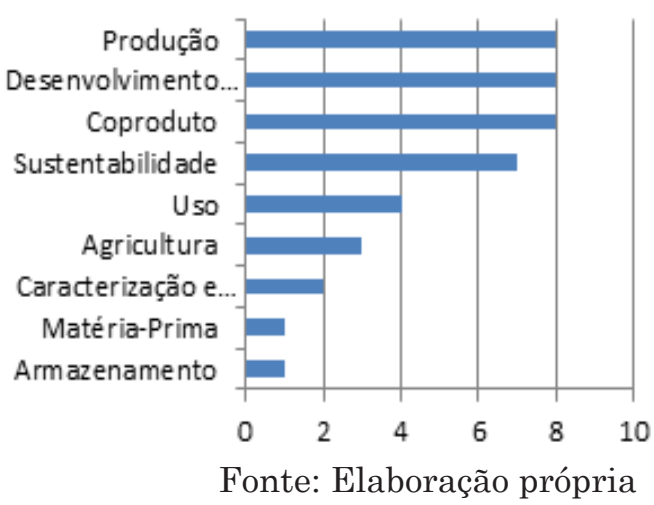

Dos 26 grupos analisados, apenas 6 afirmam possuir interações com o Setor Produtivo, perfazendo um total de 13, conforme figura 8.

A fraca interação com o setor produtivo evidencia que a ciência produzida no Brasil ainda encontra barreiras para se transformar em produtos inovadores que beneficiem a sociedade. 


\section{Figura 8 - Grupos com interação com o setor produtivo}

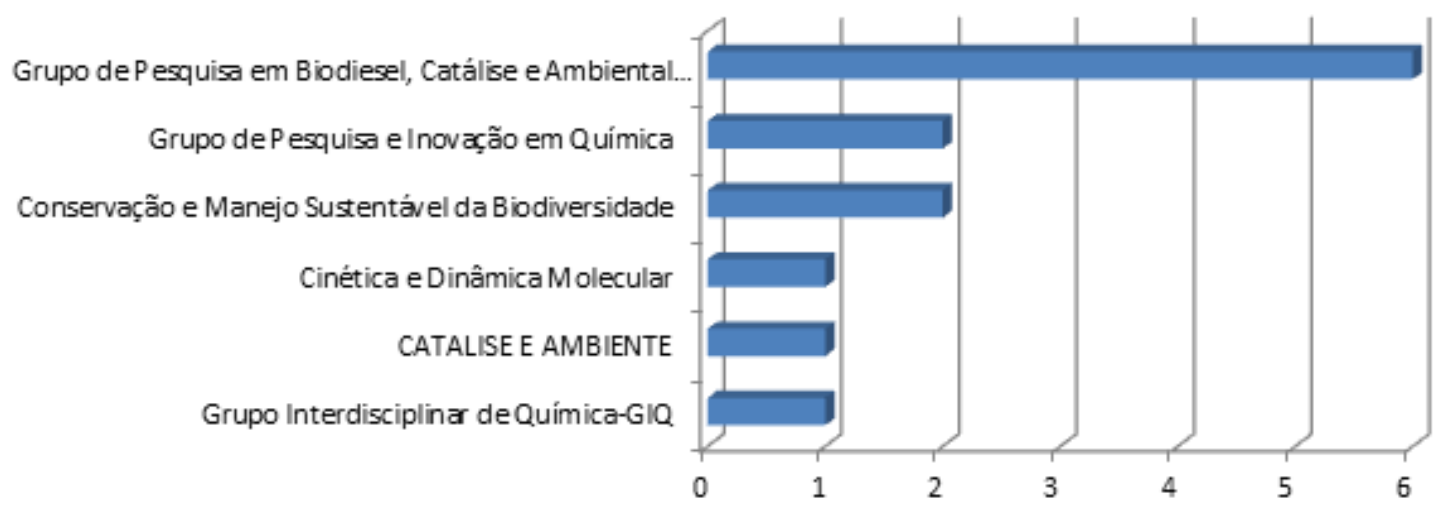

Fonte: Elaboração própria

\section{Conclusão}

O trabalho buscou mapear e produzir indicadores científicos e tecnológicos em biodiesel na Bahia. Para tanto, utilizaram-se informações oficiais disponibilizadas na base de currículos da Plataforma Lattes, nos Diretório dos Grupos de Pesquisa do Conselho Nacional de Desenvolvimento Científico e Tecnológico (CNPq) e nos anais dos Congressos da Rede Brasileira Tecnológica do Biodiesel.

Apesar das limitações existentes na metodologia utilizada, os dados levantados esperam que os indicadores aqui apresentados possam auxiliar a elaboração de políticas públicas que venham a fortalecer a inserção do biodiesel no estado da Bahia.

Como sugestão de trabalho futuro, ao final de cada biênio, que neste caso é o ano de 2013, será feito outro levantamento e atualização de todos estes indicadores.

A idéia de uma composição de apoio em P\&D\&I não garante por si só o direcionamento das diversas ações. Na Bahia e demais estados brasileiros devemse criar formas de integração e validação das ações de modo integrado, tanto do ponto de vista científico e tecnológico gerado, como das questões ambientais e sociais, sempre apoiadas em uma postura ética e abertas à sociedade de maneira geral.

\section{Referências}

Agência Nacional de Petróleo, Gás e Biocombustível - ANP. (Junho de 2013) Boletim Mensal de Biodiesel. Disponível em:< http://www.anp.gov.br/?pg=53049 \&m=\&t1 $=\& \mathrm{t} 2=\& \mathrm{t} 3=\& \mathrm{t} 4=\& \mathrm{ar}=\& \mathrm{ps}=\&$ cachebust $=1310522958651>$. Acesso em set. 2012 . 
. (2013). Dados estatísticos mensais. Disponível em: <http://www.anp. gov.br $/$ pg $=58457 \& \mathrm{~m}=\& \mathrm{t} 1=\& \mathrm{t} 2=\& \mathrm{t} 3=\& \mathrm{t} 4=\& \mathrm{ar}=\& \mathrm{ps}=\&$ cachebust $=1322573564293>$. Acesso em jun. 2013.

BAHIA. Plano de Desenvolvimento Bahia 2023. PPA 2012-2015. Disponível em:<http://ebookbrowse.com/bahia-2023-ppa-2012-2015-programas-tematicosppt-d120860301>. Acesso em set. 2012.

. Secretaria de Infraestrutura do Estado da Bahia - SEINFRA. Balanço Energético da Bahia 2010-Série 1993-2009. Disponível em:<: http://www.seinfra. ba.gov.br/balanco_2010/BEEBA2010_VP.pdf>. Acesso em set.2012.

BIODIESELBR. Vantagens do Biodiesel. Disponível em: <http://www.biodieselbr.com/ biodiesel/vantagens/vantagens-biodiesel. ht. Acesso em: set. 2012.

Brasil. Lei No 11.097, de 13 de janeiro de 2005. Dispõe sobre a introdução do biodiesel na matriz energética brasileira; altera as Leis nos 9.478, de 6 de agosto de 1997, 9.847, de 26 de outubro de 1999 e 10.636, de 30 de dezembro de 2002; e dá outras providências.Disponível em:< http://www.planalto.gov.br/ccivil_03/_ato20042006/2005/Lei/L11097.htm>. Acesso em jan. 2013.

Conselho Nacional de Desenvolvimento Científico e Tecnológico (CNPq). Grupos de Pesquisa. Disponível em:<http://www.cnpq.br/gpesq/apresentacao.htm>. Acesso em set.2012.

Liberal C.G. (2005). Indicadores de ciência e tecnologia: conceitos e elementos históricos. In: CIÊNCIA \& OPINIÃO. Curitiba, v. 2, n. 1/2, jan./dez.

Menezes R. Pesquisa, Desenvolvimento \& Inovação na Cadeia Produtiva do Biodiesel Desenvolvimento Tecnológico do Programa Nacional de Produção e Uso de Biodiesel (PNPB). Rede Brasileira de Tecnologia do Biodiesel. Disponível em: <http://www.abq.org.br/biocom/2011/trabalhos.html>. Acesso em: jun.2013.

Ministério De Minas E Energia - MME. (fev. 2013). Boletim mensal dos combustíveis Renováveis, Brasília, SPG, n. 61. Disponível em: <http://www.mme.gov.br/spg/ menu/ publicacoes.html>. Acesso em: 15 jun. 2013.

. Balanço Energético Nacional 2012. Gráficos do BEN 2012 Disponível e $\mathrm{m}:<\mathrm{http} / / / \mathrm{www} . \mathrm{mme} . g o v . b r / m m e / m e n u / t o d a s \_p u b l i c a c o e s . h t m l .>$. Acesso em maio 2013.

OCDE, Organização para Cooperação e Desenvolvimento Econômico. Manual de Oslo: diretrizes para coleta e interpretação de dados sobre inovação. (2005). Disponível em: <www.mct.gov.br/index. php/content/view/44912.html>. Acesso em out. 2012.

Parente, E. J. S. (2003). "Biodiesel: uma aventura tecnológica num país engraçado." Tecbio, Fortaleza, CE. 68p.

Portal Do Biodiesel. Biodiesel. Disponível em:<http://www.biodiesel.gov.br $>$. Acesso em ago.2012.

Portela, H. E. Avaliação Técnico-Econômica de Um Empreendimento Produtivo de Biodiesel. Dissertação (Mestrado Profissional em Gerenciamento e Tecnologias Ambientais no Processo Produtivo). Salvador, 2008. Universidade Federal da Bahia. 
Quintella, C.M. (2009). "Cadeia do biodiesel da bancada à indústria: uma visão geral com prospecção de tarefas e oportunidades para P\&D\&I.” Química Nova, São Paulo-SP, v. 32, p. 793-808.

Rede Brasileira De Tecnologia Em Biodiesel - RBTB. Ações em Desenvolvimento. Disponível em:< http://www.mme.gov.br/programas/biodiesel/menu/rede_ brasileira_tecnologia/sobre_a_rede>. Acesso em set.2012.

Silva, M. Biodiesel na Bahia: Sugestões de Ações Indutoras. Disponível em:< http:// dialogos.ftc.br/index.php?option=com_content\&task=view\&id=202\&Itemid=4>. Acesso em set.2012.

Tigre, P. B. (2006). Gestão da Inovação - A Economia da Tecnologia no Brasil.. Rio de Janeiro: Elsevier.

Winter, E. Fapesb sedia palestra sobre Indicadores Científicos, Tecnológicos e de Inovação no Brasil. Disponível em:<http://www.fapesb.ba.gov.br/?p=6510>. Acesso em set.2012. 